\section{Desarrollo de competencias en atención primaria de salud}

\author{
Carme Nebot Adell, ${ }^{1}$ \\ Carlos Rosales Echevarria ${ }^{2}$ \\ y Rosa M. Borrell Bentz ${ }^{3}$
}

Forma de citar: Nebot Adell C, Rosales Echevarria C, Borrell Bentz RM. Curso virtual para el desarrollo de competencias en atención primaria de salud. Rev Panam Salud Publica. 2009; 26(2):176-83.

Palabras clave: atención primaria de salud; atención médica; educación basada en competencias; cursos de capacitación; servicios de salud; educación a distancia; América Latina.

\footnotetext{
1 Organización Panamericana de la Salud (OPS/OMS), Asesora Regional de Servicios de Atención Primaria. La correspondencia se debe dirigir a: Carme Nebot. Avinguda America 22B, Cerdanyola del Valles, 08290, España. Correo electrónico: cnebot.bcn.ics@ gencat.cat

2 OPS/OMS, Asesor Regional de Recursos Humanos en Salud

OPS/OMS, Asesora Regional de Educación Continuada.
}

Han pasado más de 30 años desde que se introdujo ${ }^{4}$ en Alma Ata el concepto de atención primaria de salud (APS), una iniciativa dirigida a mejorar la equidad en el acceso a los servicios y la eficiencia en el uso de los recursos de salud, con un fuerte énfasis en la atención preventiva y en las poblaciones menos favorecidas. Una de sus premisas era que en muchos casos las causas de las enfermedades trascienden al sector de la salud y por lo tanto deben abordarse con un enfoque en el cual participe toda la sociedad (1).

Si bien desde entonces la salud en las Américas ha registrado progresos significativos, particularmente en la lucha contra las enfermedades y en la esperanza de vida, los sistemas de salud de la Región todavía no se han fortalecido ni extendido lo suficiente, ni han logrado la eficacia necesaria como para cumplir los objetivos de APS fijados en Alma Ata.

Ante esta realidad, en los últimos años la Organización Panamericana de la Salud ha venido analizando y evaluando un vasto volumen de información teórica y empírica sobre el alcance, el funcionamiento y la eficacia de la APS. Sus hallazgos ponen en evidencia que las razones que no permitieron una implantación más amplia y profunda del modelo responden a limitaciones que incluyen desorganización, falta de políticas de incentivos, financiamiento y costos elevados, distribución desigual de servicios y, fundamentalmente, carencia de recursos humanos capacitados y dedicados a la atención primaria. El resultado de dicha evaluación evidencia la necesidad de fortalecer y extender la atención primaria de modo tal que se convierta en el eje articulador del sistema de salud.

En este sentido, uno de los retos clave que se presentan es el de dotar a los sistemas de salud con recursos humanos en número suficiente y adecuadamente capacitados, de forma que sea el lugar donde se puedan resolver la mayoría de los problemas sanitarios que padece la comunidad.

En efecto, la renovación de la APS exige una transformación profunda en los programas de formación de los profesionales de la salud, así como la expansión y diseminación del conocimiento sobre este nivel de atención (2). Tal propósito supone resolver problemas difíciles, entre los que cabe destacar la insuficiencia de personal calificado para proveer la cobertura universal, el desequilibrio de recursos en favor de la concentración en ciudades y hospitales, la falta

\footnotetext{
4 Conferencia Internacional sobre Atención Primaria de Salud de Alma-Ata, realizada en Kazajistán, del 6 al 12 de septiembre de 1978, organizada por la OMS/OPS y UNICEF, y patrocinada por la entonces URSS.
} 
de políticas de incentivos, los elevados costos del personal, la escasez de supervisión apropiada, la formación con enfoque predominantemente curativo y orientada a las especialidades, así como el débil desarrollo del trabajo en equipo, todos considerados como los problemas más frecuentes que afrontan actualmente los servicios de salud (3-6).

En consecuencia, el avance de la estrategia de renovación comportará la necesidad de contar con más profesionales con formación específica, es decir, capacitados para desempeñar un conjunto de funciones específicas de la atención primaria. Este conjunto de conocimientos y habilidades se diferencia de los del resto del sistema y se deberá adecuar al nuevo contexto configurado conforme a la estrategia de la APS (7). Asimismo, el énfasis que dicha estrategia ejerce sobre la equidad de acceso y la calidad de los servicios requiere sistematizar los procedimientos y el trabajo en red, como poderosos instrumentos para aumentar la capacidad resolutiva de los equipos al fomentar la coparticipación y la corresponsabilidad de sus integrantes (8).

En 2005, durante una Consulta Regional celebrada en Montevideo, se definieron las líneas de acción para avanzar en el desarrollo de la estrategia de renovación de la APS (9). Una de ellas especificaba "[la necesidad de] continuar la colaboración con los países para mejorar la capacitación de los trabajadores de salud, entre ellos los que hacen las políticas y los gestores en las áreas prioritarias de la APS." Con base en esta declaración, se consideró necesario el trabajo conjunto desde la perspectiva de recursos humanos y de los servicios de salud en el abordaje de la organización, estructura y perfiles de actuación de los equipos de APS. Con la definición de las competencias, se inició la construcción de un proceso de aprendizaje dirigido a aumentar la capacidad de respuesta de los equipos de APS en la Región. Tras este primer paso, en colaboración con un grupo de expertos se diseñó un curso virtual para líderes y gestores de APS en la plataforma virtual de salud pública. La iniciativa tuvo una amplia convocatoria entre los profesionales de la salud y el curso empezó en junio de 2008, con acceso a todos los países de habla hispana de la región.

En el presente trabajo se describen las acciones llevadas a cabo por la OPS/OMS para preparar una estrategia educativa dirigida a fortalecer la APS en las Américas. Los objetivos de dicha estrategia son identificar competencias en atención primaria y diseñar un plan pedagógico que permita desarrollar o fortalecer dichas competencias.

\section{¿POR QUÉ LAS COMPETENCIAS?}

Toda renovación sistémica en los servicios de salud conlleva profundas transformaciones en los modelos de gestión y de atención, generando forzosamente demanda de nuevas competencias y modificando en consecuencia la estructura de los recursos humanos. Uno de los elementos definitorios de la educación permanente es su orientación hacia y desde el trabajo cotidiano, la programación del aprendizaje desde la realidad laboral y la orientación hacia la mejora del desempeño utilizando el enfoque de competencias profesionales. Sin duda las competencias constituyen en la actualidad una conceptualización y un modo de operar en la gestión de recursos humanos que permiten mejorar la articulación entre gestión, trabajo y educación $(10,11)$. Del análisis de sus diversas definiciones puede concluirse que las competencias son características permanentes - conocimientos, actitudes y habilidades - que la persona demuestra cuando realiza con éxito una actividad, sea laboral o de otra índole (12-17). Las principales competencias necesarias para los profesionales de los equipos de APS se pueden agrupar en seis categorías, tal como se presentan en el cuadro 1.

El curso virtual, denominado "Desarrollo de capacidades para la renovación de la Atención Primaria de Salud", se gestó a partir de la identificación de las competencias de los equipos de APS en los sistemas y servicios de salud como un componente crítico para el avance de la renovación de la atención primaria, por entenderse que la consecución de servicios de calidad, sostenibles y aceptados por la población requiere profesionales que lideren el proceso de cambio en todos los niveles del sistema.

CUADRO 1. Competencias consideradas más idóneas para la atención primaria de salud (APS)

Características específicas de la APS

Enfoque integral: vida, género, culturas, individuo, familia, comunidad Trabajo en equipo

Coordinación entre niveles: sistemas de referencia y contra-referencia Área clínica

Trato al paciente

Atención centrada en el individuo y la familia

Atención preventiva, curativa, rehabilitadora y cuidados paliativos

Seguimiento compartido

Emergencias

Atención domiciliaria

Comunicación

Entre profesionales

Entre profesionales de salud e individuos

Con la comunidad

Manejo de conflictos

Anuncio de malas noticias

Gestión del conocimiento

Uso de sistemas de información

Uso de internet

Medicina basada en pruebas (guías clínicas)

Salud pública y promoción de la salud

Conocimientos de epidemiología

Enfermedades de declaración obligatoria, brotes, campañas de vacunación

Hábitos y estilos de vida

Área de gestión

Funciones administrativas y gerenciales: liderazgo, supervisión y coordinación

Negociación

Gestión de la calidad, reclamos

Planificación y evaluación

Gestión clínica

Fuentes: referencias 18-25. 


\section{MÉTODOS}

La planificación y el diseño del curso contaron con la participación de grupos de consenso clave, y se desarrolló en dos fases que incluyeron la identificación de competencias y su aplicación al programa de capacitación.

\section{IDENTIFICACIÓN DE COMPETENCIAS}

A partir de los elementos esenciales de los sistemas de salud basados en la atención primaria según los describe el documento de posición en APS de la OPS/OMS (cuadro 2) (9), un panel de expertos internacionales reunido en junio de 2006 definió las competencias básicas necesarias para formar un equipo multidisciplinario. La selección de los miembros se basó en dos criterios centrales: su experiencia profesional y la composición de un grupo multidisciplinario. El equipo estuvo integrado por un total de 20 profesionales pertenecientes a ministerios de salud, universidades, asociaciones profesionales, organizaciones internacionales y servicios de salud de los países de la Región de las Américas.

El proceso se inició con la revisión de los elementos esenciales con que debía contar la nueva estrategia, y continuó con una lluvia de ideas orientada a identificar los conocimientos y habilidades requeridos para que la APS pueda dar una respuesta adecuada a las necesidades de la población. Tras este análisis se elaboró una matriz de competencias que posteriormente fue examinada por el mismo panel de expertos.

En septiembre de 2006 la matriz de competencias se sometió a revisión por un segundo grupo de expertos, que perfiló las bases para diseñar un plan de fortalecimiento de las capacidades de los equipos de APS, entendiendo el proyecto educativo como parte fundamental de una estrategia de cooperación internacional destinada a potenciar la atención primaria en la Región de las Américas. Los 15 participantes de este segundo grupo fueron elegidos bajo los mismos criterios, incluyendo profesionales con reconocido prestigio internacional por su consolidada trayectoria en la salud pública y en la APS.

En el cuadro 3 se presentan las competencias consideradas como esenciales para los equipos de APS.

CUADRO 2. Elementos esenciales de los sistemas de salud basados en la atención primaria de salud

Acceso y cobertura universal

Atención integral, integrada y continua

Énfasis en promover la salud y prevenir la enfermedad

Orientación familiar y comunitaria

Mecanismos activos de participación

Marco político, legal e institucional sólidos

Organización y gestión optimas

Políticas y programas pro-equidad

Primer contacto

Recursos humanos apropiados y sostenibles

Acciones intersectoriales

Fuente: referencia 9

\section{DISEÑO DEL CURSO}

Una vez completada la matriz de competencias, durante el último trimestre de 2006 se definieron cinco líneas temáticas a partir de las cuales se construyeron los módulos, ordenándose los correspondientes conocimientos y habilidades específicos para cada una de las competencias requeridas: enfoque intersectorial, enfoque integral, orientación familiar y comunitaria, gestión, y solidaridad y equidad en los servicios.

En marzo de 2007 se convocó un nuevo panel de 30 profesionales especialistas en servicios de salud, salud internacional y salud pública, con el mandato de determinar los objetivos y el alcance del programa educativo, desarrollar la propuesta temática del curso y definir su estructura y metodología, revisar la bibliografía de apoyo y organizar el plan de trabajo para la elaboración del curso. A continuación se formuló la propuesta de estrategia educativa, que además del curso virtual incluye el desarrollo de proyectos locales de capacitación y la generación de una base de datos bibliográficos sobre APS. En la figura 1 se sintetizan las fases seguidas hasta la finalización de la propuesta educativa.

\section{EL PRODUCTO}

La identificación de competencias constituyó un valioso elemento para diseñar un programa que considera el aprendizaje y la práctica diaria como componentes clave de la nueva estrategia de APS. Este enfoque retoma las orientaciones básicas de la educación permanente, que promueve el aprendizaje a lo largo de la vida y lo considera como un proceso que se alimenta a través del intercambio de experiencias, información y conocimiento.

En febrero de 2008 finalizó el proceso que incluyó la definición del enfoque educativo, el diseño de la propuesta, el ajuste de los módulos, la edición de los materiales, la selección de la bibliografía y la formulación de los mecanismos de evaluación. El curso virtual sobre desarrollo de capacidades para la renovación de la APS estaba listo para ser puesto en marcha en el marco de una estrategia de cooperación técnica con los países de la Región.

La aplicación extensiva del curso considera a los socios académicos, como universidades y centros docentes, y a los nodos del Campus Virtual de Salud Pública (CVSP) de los países de la Región de las Américas, como agentes imprescindibles para la formación y la capacitación de los equipos de APS. Estas entidades adecuarían sus actividades conforme a las prioridades y necesidades de cada contexto nacional, mientras que la OPS/OMS actuaría como organismo facilitador del proceso de aplicación en los países.

La selección de los tutores, efectuada con el respaldo de los nodos del CVSP, se llevó a cabo atendiendo a los siguientes criterios: a) debían ser profesionales con formación y experiencia en docencia $\mathrm{y}$ en servicios de salud, y tener conocimiento de la 


\section{CUADRO 3. Competencias esenciales de los equipos de atención primaria de salud (APS)}

\begin{tabular}{l} 
Conocimientos \\
\hline Identificación de barreras de acceso \\
Reconocimiento y análisis de actores \\
Estrategias para alcanzar la cobertura universal \\
Atención integral, integrada y continua \\
Técnicas y procedimientos para la atención intra y extramural \\
Mecanismos de referencia y contrarreferencia \\
Ciclo vital individual y familiar \\
Situación de salud de la comunidad, sus determinantes y \\
condicionantes \\
Promoción de la salud y prevención de la enfermedad
\end{tabular}

Buenas prácticas

Nuevas tecnologías

Uso de la red de servicios de atención

Identificación y definición de necesidades de servicios

Priorización de intervenciones

Identificación de actores e instituciones en la comunidad Comunicación entre los miembros del equipo y la comunidad

Políticas públicas y marcos legales vinculados con el sector salud Identificación de grupos de poder locales

Nociones de gestión administrativa

Identificación de organizaciones sociales, gremiales y sus actores Registros específicos en APS

Identificación y análisis de inequidades existentes

Resolución de inequidades en salud, marcos políticos y legales

Proceso de acogida al individuo/familia.

Entrevista clínica

Identificación y resolución de problemas

Conocimiento y definición de roles y responsabilidades dentro del equipo

Código ético de los trabajadores de la salud

Identificación de recursos necesarios

Identificación de sectores vinculados a la salud

Definición de acciones intersectoriales

Prevención de desastres y mecanismos de respuesta en situaciones de emergencia
Habilidades para:

Aplicar estrategias que reduzcan barreras de acceso, amplíen la cobertura y reduzcan las inequidades

Identificar necesidades percibidas y no percibidas por el individuo y la familia

Abordar el ciclo vital

Promover el autocuidado de la salud en la comunidad, según las enfermedades prevalentes

Promover el aprendizaje sobre la APS por parte del equipo de salud y la comunidad

Establecer compromisos o alianzas para la producción social de la salud

Liderar y participar en las iniciativas de promoción de la salud y prevención de la enfermedad para la comunidad

Brindar atención apropiada, basada en la evidencia en distintos escenarios

Aplicar los procedimientos de manera oportuna y eficiente

Definir las responsabilidades de cada uno de los miembros del equipo

Identificar fuentes de información y actores clave en la comunidad Recolectar, analizar y utilizar la información adecuada y pertinente Identificar y definir las necesidades de salud de la comunidad

Establecer prioridades de intervención

Estimular la participación activa de la comunidad en la gestión del sector salud.

Optimizar la información, comunicación y educación

Analizar la coherencia entre las políticas públicas y el trabajo multidisciplinar en APS

Propiciar el desarrollo de políticas locales a través de la participación ciudadana

Desarrollar planificación estratégica, investigación operativa y evaluación del desempeño

Seguir el proceso de gestión administrativa

Involucrar $a-y$ trabajar activamente con - los líderes reconocidos

Identificar y corregir inequidades a través del desarrollo de políticas y programas

Incorporar la participación social en la resolución de las inequidades

Brindar un trato digno y respetuoso

Acoger al individuo dentro del sistema de salud

Desarrollar una buena comunicación, a nivel individual y colectivo

Identificar problemas que repercuten en el proceso salud-enfermedad

Apoyar y participar en el proceso de formación permanente de los miembros del equipo

Elaborar, desarrollar y participar en procesos del trabajo en equipo: comunicación, colaboración, coordinación, negociación y solución de conflictos, participación, toma de decisiones

Hacer un uso racional y eficiente de los recursos disponibles Presentar información útil y detallada a los tomadores de decisión

Liderar, participar y apoyar las acciones intersectoriales

Movilizar recursos intersectoriales y de la propia comunidad para responder a las necesidades de salud

Interactuar con las autoridades nacionales y la comunidad

Transmitir las informaciones requeridas e identificar rutas de evacuación, lugares seguros, refugios

Fuente: Paneles de expertos OPS/OMS en Tegucigalpa, Honduras, y Fortaleza, Brasil. Junio y septiembre de 2006. 
FIGURA 1. Desarrollo del curso virtual sobre atención primaria de salud, fases y componentes esenciales

Fases del proceso

\begin{tabular}{|l|}
\hline $\begin{array}{l}\text { Junio 2006 } \\
\text { Panel de expertos }\end{array}$ \\
\hline $\begin{array}{l}\text { Septiembre 2006 } \\
\text { Panel de expertos }\end{array}$ \\
\hline Último trimestre 2006
\end{tabular}

Inicio del diseño

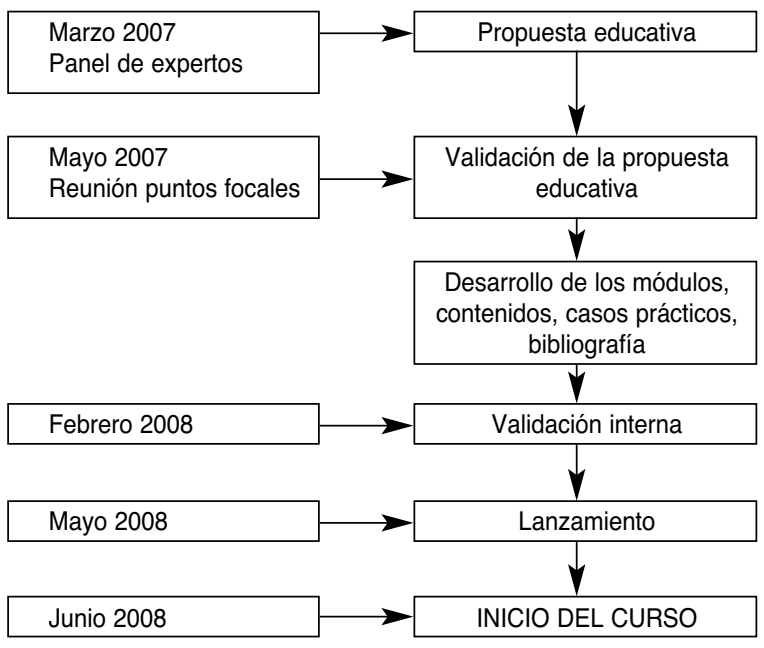

Fuente: OPS/OMS. Área de sistemas y servicios de salud. Washington DC. 2008.

estrategia de renovación de la APS, así como de la filosofía emanada de la Conferencia de Alma Ata, además de su disponibilidad para guiar a los estudiantes a lo largo de todo el curso. Cada país seleccionó a cuatro tutores provenientes del Ministerio de Salud, la seguridad social y de universidades y otros centros académicos. Todos asistieron a un taller para ayudarlos a familiarizarse con el curso y con la plataforma educativa Moodle, insertada en el CVSP, así como para analizar sus potencialidades como ejecutores efectivos de las clases virtuales.

El curso virtual de APS, con una duración de 27 semanas y 160 horas lectivas, está estructurado en módulos organizados por unidades temáticas estrechamente relacionadas y en forma secuencial (figura 2). Sus contenidos abordan aspectos conceptuales relevantes, desafíos prioritarios del momento actual y ejercicios de solución de problemas relacionados con la APS. Paralelamente, con el fin de reforzar la aplicación práctica de los contenidos aprendidos, a partir del primer módulo los participantes comienzan a diseñar de manera secuencial un proyecto de intervención. En el último módulo se propone un ejercicio final de integración de los conocimientos adquiridos y la formulación de un proyecto de intervención dirigido a desa-
FIGURA 2. Estructura y contenidos del curso virtual de desarrollo de capacidades para la renovación de la atención primaria de salud (APS)

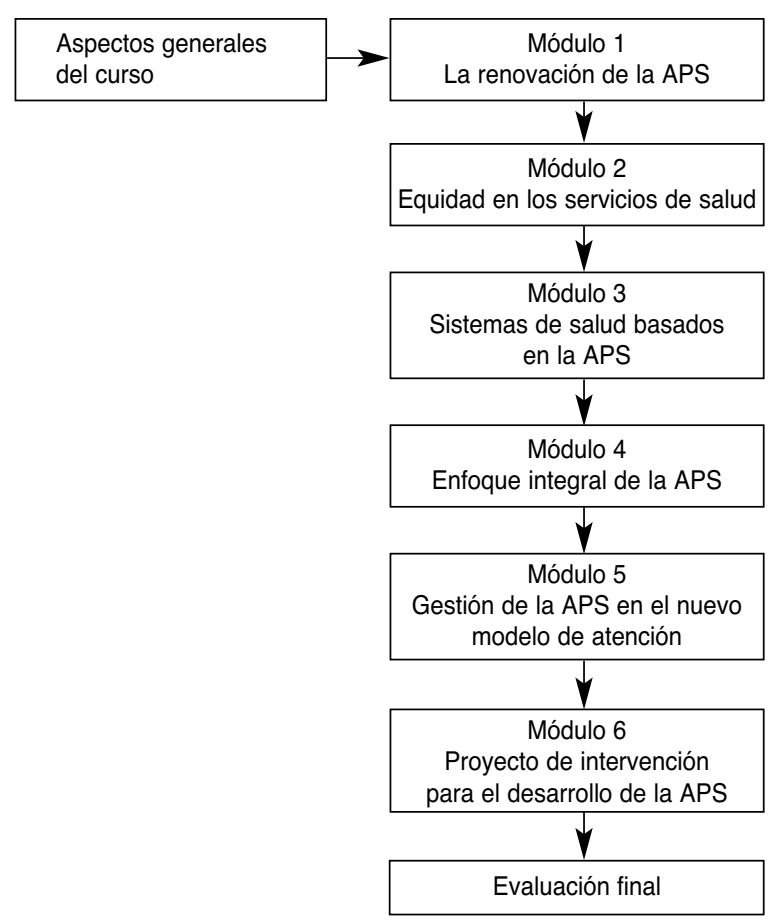

Fuente: OPS/OMS. Área de sistemas y servicios de salud. Washington DC. 2008.

rrollar la estrategia de APS renovada en el contexto nacional de cada participante.

Los resultados esperados del curso son:

- Incrementar las competencias de los miembros de los equipos multidisciplinares de APS para responder a las necesidades de salud en áreas poco pobladas —excluidas de la atención primaria- de los países de la Región.

- Desarrollar estrategias de aprendizaje dirigidas a los trabajadores de la salud para que generen proyectos de intervención —organización, gestión y evaluación-que mejoren la efectividad de la APS.

- Construir una red de actores claves - profesionales de APS, gestores y académicos-y fomentar la cultura de alianzas.

- Capacitar a profesionales de la salud para que se conviertan en formadores y generadores de estrategias de aprendizaje para los equipos locales de APS en la Región.

\section{DISCUSIÓN}

La estrategia de renovación de la APS contempla la capacitación de los recursos humanos como uno de los pilares esenciales para su desarrollo, considerándose prioritario el fortalecimiento de las competencias de aquellos profesionales que deberán liderar el proceso de transformación hacia sistemas de salud basa- 
dos en la APS. El curso virtual que aquí se presenta pretende ser una herramienta para respaldar a quienes toman decisiones, en su cometido de guiar los cambios en el sector salud hacia servicios accesibles, de calidad y merecedores de la confianza de toda la ciudadanía.

Si bien este curso está dirigido exclusivamente a los tomadores de decisión, por entender que ellos son actores imprescindibles para el cambio de orientación hacia los sistemas de salud basados en la APS, en el medio plazo está previsto diseñar otro curso virtual para la capacitación de profesionales del primer nivel. La decisión de diseñar dos cursos distintos - uno para el nivel de gestión y otro para el asistencial- se adoptó durante el proceso de identificación de competencias, cuando quedó claro que primero había que capacitar a quienes son responsables de gestionar el escenario donde se proveen los servicios de atención primaria, de forma que los profesionales que prestan los servicios trabajen en condiciones más favorables y se sientan más motivados para mejorar su desempeño.

La evaluación del programa sigue los lineamientos del Campus Virtual de Salud Pública y se realiza de manera continua y sistemática, mediante la recogida y análisis de información fiable y confiable. La OPS financia los tutores que participan en la iniciativa y en un futuro también se prevé el patrocinio de varias universidades de la Región.

Dada su proximidad y relación con la comunidad, la atención primaria debe manejar correctamente y resolver los problemas de salud que le son inherentes (26). Las habilidades consideradas imprescindibles en sus recursos humanos se refieren entonces a la capacidad de trabajar en equipo, la comunicación y el enfoque integral y comunitario de los cuidados (2729). Si bien en los últimos años las universidades han registrado progresos importantes en la formación de profesionales de la salud, en el caso de la atención primaria todavía se observan marcadas insuficiencias respecto a los conocimientos y habilidades necesarios para dar respuesta a las demandas efectuadas por una comunidad cada vez más informada y exigente (30).

Tradicionalmente la APS se ha polarizado hacia la atención maternoinfantil y - predominantementehacia los casos agudos de salud, olvidando los cuidados preventivos, las enfermedades crónicas y los factores sociales que afectan a la salud. El abordaje de las necesidades de las personas, la familia y la comunidad requiere profesionales idóneos en competencias esenciales para la atención primaria, como la comunicación, razonamiento lógico, gestión, bioética, interacción con personas excluidas socialmente, violencia doméstica y otros factores sociales de riesgo para la salud (19).

El fortalecimiento del primer nivel de atención exige asimismo que los programas de capacitación incorporen conocimientos teóricos y prácticos, científicos, técnicos y humanos, y que su orientación incluya a los profesionales que toman decisiones (31). Las áreas objeto de capacitación deben centrarse en temas referidos a la solución de problemas, al manejo de la información para la toma de decisiones y a la equidad y eficiencia en la distribución de recursos (32). La comunicación, por su parte, es una competencia básica en atención primaria $(19,27)$, ya que el contacto continuado y directo brinda la oportunidad de conocer mejor a los pacientes y consolidar buenas relaciones con la comunidad $(20,33)$. La destreza para comunicarse en atención primaria implica específicamente la capacidad de los profesionales para captar los motivos de preocupación de los individuos, considerar las necesidades psicosociales e involucrar a los pacientes en las decisiones sobre su propia salud; todas ellas contribuyen a mejorar la relación de la población con los servicios (34). Así, la empatía generada por una relación duradera se ve enormemente favorecida por la capacidad de dichos profesionales para comunicarse con la población de referencia a nivel individual o colectivo. Además, el hecho de que los profesionales de la atención primaria son a menudo testigos de los eventos que acontecen a lo largo de la vida de los individuos, refuerza su capacidad para manejar situaciones emocionales especialmente delicadas o dolorosas.

Las competencias identificadas como esenciales en este proyecto coinciden con las sugeridas en la literatura por distintos autores (18-25). Sin embargo, lo que confiere coherencia al proceso descrito en este trabajo es que la matriz de competencias se definió a partir de componentes de importancia crítica en los sistemas basados en la atención primaria. Dichos componentes habían sido revisados y discutidos por los distintos grupos de expertos de la Región que participaron en este proyecto a lo largo de dos años (9). Otro valor añadido que hay que destacar es que ese proceso permitió identificar las destrezas y conocimientos necesarios para los miembros de los equipos de atención primaria. En cuanto a las actitudes, si bien fueron consideradas muy valiosas para el desempeño de los profesionales, no se analizaron en este ejercicio debido a su directa relación con el proceso de gestión, las condiciones laborales, los sistemas de evaluación del desempeño y los sistemas de incentivos existentes, aspectos que no forman parte de la presente iniciativa.

Una vez definidas las habilidades y competencias requeridas por el sistema para responder a las necesidades de la población, el siguiente paso es el diseño de los planes de capacitación para formar especialistas, profesionales, técnicos y trabajadores calificados que permitan avanzar hacia un Sistema de Salud Basado en la Atención Primaria de Salud (SSBAPS). En esta fase del proceso, se hará inestimable la participación y el patrocinio de centros docentes, universidades y escuelas de salud pública, cuyos aportes serán decisivos para definir planes de estudios, tanto de pregrado, como de posgrado, de acuerdo a los nuevos desafíos de la APS $(35,36)$. Harán falta entonces modificaciones en la orientación de la educación hacia la atención primaria, entre cuyas premisas cabe destacar la financiación suficiente para mantener centros de calidad, el desarrollo de un plan de estudios común para las distintas disciplinas y la posibilidad de que los pro- 
fesionales puedan recibir su formación en centros de atención primaria $(37,38)$. Más aún, en los últimos años se han constatado las ventajas de desplazar la formación desde las aulas al seno de la comunidad, precisamente donde los trabajadores de la salud deberán aplicar sus habilidades y conocimientos (30). Este tipo de iniciativas son consideradas fundamentales para adquirir las competencias necesarias en APS, pues permite el contacto de los profesionales con los individuos y sus familias, familiarizándolos con sus características sociales y culturales (39).

Por último, la globalización y el nuevo escenario mundial plantean el reto de actuar frente a problemas de salud hasta ahora desconocidos. En consecuencia, los profesionales de APS deberán estar capacitados para responder a la aparición de nuevas enfermedades, crisis y desastres, sean entidades conocidas o extrañas a la zona afectada, todo lo cual reclamará una respuesta efectiva de los servicios conectados en red al primer nivel (40).

\section{CONCLUSIONES}

Para que la atención primaria pueda actuar como eje articulador del sistema de salud es preciso que sus profesionales tengan una sólida formación que les permita responder oportuna y efectivamente a las necesidades de la población. Tal responsabilidad implica capacidad para trabajar en equipo, conocimientos técnicos para brindar atención médica apropiada y habilidades para comunicarse con los otros miembros del equipo y con la comunidad. Y tan importante como lo anterior, los profesionales que brindan estos servicios deben contar con líderes y tomadores de decisión que conozcan y entiendan los beneficios potenciales de la APS, no sólo en términos de mejora del estado de salud de la población, sino también contribuyendo a un funcionamiento más eficiente del sistema sanitario.

Agradecimientos. Agradecemos la colaboración de Armando Güemes, Cristianne Rocha, Michele Santana y José María Valderas. Expresamos también nuestra gratitud a Hannu Vuori, Hernan Montenegro, Liliana Arias Castillo, Yves Talbot y Jaime Gofin, por sus valiosos comentarios a los distintos contenidos del proyecto.

\section{SYNOPSIS}

\section{Development of primary health care competencies}

Given that the lack of qualified human resources devoted to primary health care (PHC) is one of the reasons why Alma Ata goals are as yet unattained, PAHO/WHO sponsored the design and implementation of a virtual course for training health care professionals at this level. The course was designed around competencies identified as being necessary for renewing primary care, founded on the premise that providing quality, sustainable services amenable to the public must emanate from professionals who lead the change process at all levels in the system. In this first phase, instruction is aimed at PHC leaders, managers, and other decision makers. The course is offered through the Virtual Public Health Campus and is 27 weeks long.

Keywords: Primary health care; medical care; competency-based education; training courses; health services; distance education; Latin America.

\section{REFERENCIAS}

1. Organización Mundial de la Salud. Informe sobre la salud en el mundo 2008. Ginebra: OMS; 2008.

2. Showstack J, Lurie N, Larson E, Rothman AA, Hassmiller S. Primary care: the next Renaissance. Ann Int Med. 2003; 138(3):268-73

3. Pan American Health Organization. Gestión descentralizada de recursos humanos de salud en la reforma sectorial. En: Brito P, Campos F, Novick M, eds. Gestión de recursos humanos en las reformas sectoriales en salud: cambios y oportunidades. Washington, DC: PAHO; 1996. Pp 3-22.

4. Magnussen L, Ehiri J, Jolly P. Comprehensive Versus Selective Primary Health Care: Lessons For Global Health Policy. Health Affairs. 2004;23(3):16776. http://content.healthaffairs.org/ cgi/reprint/23/3/167. Acceso el 23 septiembre 2008 .
5. Yarnall KS, Pollak KI, Ostbye T, Krause KM, Michener JL. Primary care: is there enough time for prevention? American Journal of Public Health. 2003;93(4): $635-41$.

6. Sociedad Andaluza de Medicina Familiar y Comunitaria. Evaluación del modelo de reforma de atención primaria en Andalucía. Conclusiones de la revisión documental y evaluación estratégica de la reforma de atención primaria. Jaén, España, 1994.

7. Molina-Durán F, Ballesteros-Pérez AM, Martínez-Ros MT, Soto-Calpe R, Sánchez-Sánchez F. Perfil profesional del personal sanitario en atención primaria. Un estudio Delphi. Aten Primaria. 1996; 17(1):24-33.

8. Aguirre-Huacuja E. La corresponsabilidad operativa en la atención primaria a la salud. Salud Pub Mex. 1994;36(2): 210-13.
9. Organización Panamericana de la Salud/ Organización Mundial de la Salud. La renovación de la atención primaria de salud en las Américas. Documento de Posición de la Organización Panamericana de la Salud; Washington DC, OPS/ OMS, 2007.

10. Irigoin $M$, Vargas Zuñiga F. Competencia laboral: Manual de conceptos, métodos y aplicaciones en el sector de la salud. Montevideo, Centro Interamericano para el Desarrollo del Conocimiento en la Formación Profesional; 2002. p. 252.

11. Irigoin $M$, Vargas Zuñiga F. Certificación de competencias: del concepto a los sistemas. Boletín Técnico Interamericano de Formación Profesional. Montevideo. 2002;152:75-88.

12. Organización Internacional del Trabajo/ Centro Interamericano para el Desarrollo del Conocimiento en la Formación 
Profesional. Rodríguez Trujillo N. ¿Qué son las competencias? Universidad Central de Venezuela. Psico Consult CA http://www.ilo.org/public/spanish/ region/ampro/cinterfor/temas/ complab/doc/otros/sel_efe/i.htm Acceso el 13 de octubre de 2008.

13. Tejada Fernandez J, Navio Gamez A. El desarrollo y la gestión de competencias profesionales: una mirada desde la formación. Revista Iberoamericana de Educación. 2005;37(2):1-15. Versión digital. Acceso el 15 de octubre de 2008.

14. Villalbí JR, Aboal XL, Gonzalez-Alonso J. Los servicios de salud pública: progresos y problemas prioritarios. En: JM Cabasés, JR Villalbí, C Aibar, eds. Informe SESPAS 2002: Invertir para la salud. Prioridades en salud pública. Valencia: Escuela Valenciana de Estudios para la salud; 2002. Pp. 545-64.

15. Boletín Oficial del Estado. Ley 16/2003, de Cohesión y calidad del Sistema Nacional de Salud. BOE. 29 de mayo de 2003.

16. Martin Moreno JM. Hacia un modelo de cooperación y armonización en el campo de la salud pública en España. Rev Esp Salud Publica. 2002;76(6):637-43.

17. Sociedad Española de Medicina Familiar y Comunitaria. Portfolio. http:// www.dpisemfyc.com/publico/info/ portfolioIntegral/seccion1.jsp. Acceso el 23 septiembre de 2008.

18. Martín Zurro A, Cano Pérez JF. Atención primaria. Conceptos, organización y práctica clínica. 3a Edición. Barcelona: Doyma Libros SA; 1994.

19. Bucholtz JR, Matheny SC, Pugno PA, David A, Bliss E B, Korin EC. Task Force Report 2 of the Task Force on Medical Education. Annals of Family Medicine. 2004;2:S51-S64. http://www.annfam med.org/cgi/content/full/2/suppl_1/ s51? maxtoshow $=\&$ HITS $=10$ \& hits $=10 \&$ RESULTFORMAT=\&author1=Bucholtz + JR\&andorexactfulltext $=$ and\&searchid $=$ $1 \&$ FIRSTINDEX $=0 \&$ sortspec $=$ relevance \&resourcetype=HWCIT. Acceso el 13 octubre de 2008.

20. Guerra de la Torre G. La implantación y el desarrollo del nuevo programa de la especialidad de medicina familiar y co- munitaria. Una nueva oportunidad. Editorial. Aten Primaria. 2005;35(3):119-20.

21. Merckaert I, Libert Y, Razavi D. Communication skills training in cancer care: where are we and where are we going? Curr Opin Oncol. 2005;17(4):319-30.

22. Marchal F, Dravet F, Classe JM, Campion L, Francois T, Labbe D, et al. Postoperative care and patient satisfaction after ambulatory surgery for breast cancer patients. Eur J Surg Oncol. 2005; 31(5):495-9.

23. Weiner SJ, Barnet B, Cheng TL, Daaleman TP. Processes for Effective Communication in Primary Care. The Future of Generalism in Medicine. The Annals of Internal Medicine. American College of Physicians. 2005;142(8):709-14.

24. Leong SL, Gingrich D, Lewis PR, Mauger DT, George JH. Enhancing doctorpatient communication using email: a pilot study. J Am Board Fam Pract. 2005; 18(3):180-8.

25. World Health Organization Europe Fourth Meeting of the ST Petersburg initiative for education and training in general practice and family medicine. Report on a Meeting Minsk, Belarus, 12-14 October 2000. Copenhagen, Denmark: WHO Europe; 2001.

26. Caminal J, Starfield B, Sanchez E, Casanova C, Morales M. The role of primary care in preventing ambulatory care sensitive conditions. Eur J Public Health. 2004;14(3):246-51.

27. Kekki P. Training for primary health care. World Health Forum. 1989;10:393-6.

28. World Health Organization. Health Systems: principled integrated care. In: The World Health Organization 2003Shaping the Future. Chapter 7. Geneva: WHO; 2003.

29. Stille CJ, Jerant A, Bell D, Meltzer D, Elmore JG. Coordinating Care across Diseases, Settomgs, and Clinicians: A key Role for the Generalist in Practice. The Future of Generalism in Medicine. The Annals of Internal Medicine. American College of Physicians. 2005;142(8 supl):700-8.

30. Rivers K, Aggleton P, Whitty G. Professional development in health promotion. Health Education Authority, London, England; 1998.
31. Gervás J. La gerencia vista desde la consulta, a través del prisma de la calidad asistencial. Sociedad Española de Médicos de Atención Primaria. SEMERGEN. 2005;31(1)15-7.

32. Banerii D. Alma-Ata showed the route to effective resource allocations for health. Bull World Health Organ. 2004; 82(9):707-8.

33. Bredart A, Bouleuc C, Dolbeault S. Doctor-patient communication with care in oncology. Curr Opin Oncol. 2005; 17(4):351-4.

34. Mallinger JB, Griggs JJ, Shields CG. Patient-centered care and breast cancer survivors' satisfaction with information. Patient Educ Couns. 2005;57(3):342-9.

35. Jardines JB, Aneiros-Riba R, Salas-Perea RS. Cuba: Recursos humanos en la atención primaria de salud y su estrategia de desarrollo. Educ Med Salud. 1993;27(2): $145-59$.

36. Vuori H. The role of the schools of public health in the development of primary health care. Health Policy. 1985;4(3):22130.

37. Showstack J, Lurie N, Larson E, Rothman AA, Hassmiller S. Primary care: the next Renaissance. Annals of Internal Medicine. 2003;138(3):268-73.

38. Schwartz MD, Basco WT Jr., Grey MR Elmore JG. Rekindling Student Interest in Generalist Careers. The Future of Generalism in Medicine. The Annals of Internal Medicine. American College of Physicians. 2005;142(8 supl):715-24.

39. Shoo R. Training primary health care workers to foster community participation. World Health Forum. 1991;12:55-62.

40. Alvarez MJ Pasquin, Mayer Pujadas MA, Llor Vilá C. ¿Qué papel desempeña la atención primaria en el abordaje y el control de nuevas enfermedades? Gripe aviar, síndrome respiratorio agudo grave, bioterrorismo y otras. Aten Primaria. 2005;35(4):204-7.

Manuscrito recibido el 13 de mayo de 2008. Aceptado para publicación, tras revisión, el 22 de octobre de 2008. 